\title{
Políticas para la inclusión social mediante Tecnologías de la información y la comunicación.
}

\author{
Iolanda García González \\ iolanda.garcia@ub.edu \\ Universidad de Barcelona
}

\section{Introducción}

"Una técnica no es buena, ni mala (depende de los contextos, de los usos y de los puntos de vista), ni neutra (ya que es condicionante o constreñidora, dado que aquí abre y aquí cierra el abanico de las cosas posibles). No se trata de evaluar los "impactos", si no de tener en cuenta las irreversibilidades a las cuales nos conduciría su uso, de ver las oportunidades que nos permitiría aprovechar, de formular los proyectos que explotarían las virtualidades que comporta y de decidir qué haremos con ella.” (Levy, 1998: 23)

Este artículo trata sobre aquellas medidas políticas y programas dirigidos a los jóvenes en situación de riesgo de exclusión social, que incorporan usos de las tecnologías de la información y la comunicación (TIC). A partir de la descripción de algunas formas de exclusión emergentes en la sociedad de la información, analizaremos el tipo de respuestas, en términos de políticas públicas, que generan. También intentaremos mostrar el valor añadido que las TIC aportan a este tipo de programas, específicamente dirigidos a colectivos jóvenes.

Para la documentación de este artículo hemos realizado una búsqueda y selección de medidas restringida al ámbito europeo y a iniciativas gubernamentales (aunque en muchas de ellas se suma la intervención de organizaciones sin afán de lucro, asociaciones o empresas). Debemos justificar el reducido número de referencias concretas a la franja de población objeto de estudio en este artículo, por la ausencia de documentación, específicamente relativa a personas de corta edad, en esta temática.

\section{La brecha digital. Características de una nueva forma de exclusión social.}

Existen diferentes definiciones y aproximaciones al concepto de exclusión social. En este caso, lo entendemos no solo en términos de carencias de tipo material, sino como dependiente del grado de acceso relacional y uso de los servicios disponibles en la sociedad, de la integración en redes sociales protectoras, así como del nivel de participación en esta (Marco, 2000; Arriba, 2001; La Parra y Tortosa, 2002). Por lo tanto, el concepto de exclusión social va más allá de las formas de desigualdad “clásicas” para penetrar en el terreno de la nointegración social. Así mismo, no se puede hablar de un único tipo de exclusión social, sino que existen múltiples y diversas facetas, situaciones y circunstancias desencadenantes (Castel, 1997). A pesar de su multidimensionalidad, podemos decir que la mayoría de casos de exclusión social se deben a alguno o a varios de los siguientes factores: precariedad laboral, desempleo de larga duración, pobreza, cambios en el mercado laboral, falta o debilidad de redes o conexiones familiares y sociales, migración, enfermedad estigmatizada o creación de guetos como consecuencia de cambios sociales. Estos factores se pueden resumir en tres dimensiones: disponer de una red social constituida por personas en las que confiar, como familiares, amigos cercanos, vecinos y/o compañeros; disfrutar de un cierto estatus social, que tiene que ver con el prestigio a los ojos de la sociedad; y poseer un trabajo remunerado, así como un cierto nivel de ingresos (Subirats, 2004).

Balla y Lapeyre (1997) distinguen entre la exclusión de las redes sociales, de la vida económica y de la vida política. La exclusión económica se asocia al desempleo, a bajos ingresos, y bajos niveles de acceso a ciertos bienes y servicios. La exclusión política tiene que ver con la privación de determinados derechos políticos a ciertos grupos y con la falta de participación. Por último, la dimensión social está relacionada con el nivel de acceso a los servicios sociales y las oportunidades para la participación social. 
En este caso, adoptaremos la definición aportada por Balla y Lapeyre (1997) pero añadiremos una dimensión: la dimensión educativa de la exclusión, que tiene que ver con el uso del derecho a la educación y el acceso a la formación durante toda la vida. La razón que justifica el hecho de añadir esta dimensión es que partimos de la idea que la educación constituye un requisito para el desarrollo de las capacidades que permiten acceder y utilizar los diferentes servicios, productos y bienes en general, que la sociedad pone a nuestra disposición.

Con el fin de acotar un poco más la clase de objeto de este artículo, debemos decir que a pesar del sentido práctico de las diferenciaciones realizadas por los autores aludidos, la mayor parte de aproximaciones al concepto de exclusión, coinciden al referirse a éste como un fenómeno procesal, en el cual interrelacionan los distintos tipos o facetas de la exclusión mencionados, entre otros matizables. Por otra parte, si partimos de la base de considerar el acceso al empleo como uno de los factores clave para la inclusión social, nos damos cuenta de que, en el caso de aquellas personas que no han alcanzado la edad madurativa para trabajar, resulta incorrecto hablar de "exclusión social”. En estos casos, consideramos que es más correcto utilizar el concepto de vulnerabilidad social, en tanto que las condiciones familiares o del entorno social en el que viven algunos niños/as o adolescentes, puede hacerles más "vulnerables", o con una mayor probabilidad de penetrar en procesos de exclusión social en la edad adulta.

De cualquier modo, lo que nos gustaría aclarar es que al referirnos al concepto de exclusión lo hacemos como calificativo de una realidad potencial, más que de una realidad fáctica[1].

Llegado este punto debemos aludir al concepto de fractura o brecha digital (digital divide) el cual se refiere, en su sentido más genérico, a la distancia entre aquellas personas y comunidades que dominan, acceden y utilizan eficazmente las TIC, y aquellas que no (BECTA, 2001). En Europa, la fractura digital es evidente y las estadísticas muestran como generalmente se suma, y amplifica los factores tradicionales de riesgo de exclusión social como el bajo nivel formativo, la edad o el género, la ocupación o el nivel de ingresos. El acceso a los ordenadores y a Internet, así como la habilidad para hacer un uso eficaz y social de las TIC son ya importantes para la plena participación en la vida política, económica y social en los países desarrollados y esta tendencia va en aumento. Como mantiene Bautista (2001), un sujeto será tanto más autónomo e independiente, cuanto mayor sea su nivel de participación en el desarrollo tecnológico de la sociedad.

\section{Las TIC, como (colateral) y como remedio (complementario) de la exclusión social}

En la mayor parte de ciudades europeas, solo una pequeña parte de los ciudadanos tiene acceso a las redes informáticas y grandes proporciones de población carecen de las habilidades básicas para el uso de las TIC. La revolución tecnológica y la globalización, como fenómenos marco de la sociedad de la información se contemplan con optimismo o recelo bajo el prisma de distintos sectores. No obstante, incluso autores que se posicionan claramente a favor de la apropiación de las TIC en todos los ámbitos vitales como Castells, mantienen que la rapidez del cambio tecnológico, junto a la debilitación de la estructura de familia tradicional y la crisis del estado del bienestar, puede llevar a un incremento de la fragmentación social y la consecuente exclusión de aquellos grupos sociales más desfavorecidos, al no disponer estos de la cualificación necesaria para hacer frente a dichas transformaciones (Castells, 1997). Por otra parte, algunos autores contemplan a las TIC como medios que conducen al aislamiento, aunque diversos estudios muestran como Internet puede contribuir al desarrollo de la sociabilidad, ampliar los vínculos sociales y generar nuevos tipos de comunidades (online) o transformar los ya existentes al añadir la dimensión virtual (Wellman, Boase y Chen, 2002; Comisión Europea, 2003). Incluso el incremento de la información es contemplado como algo negativo desde la hipótesis del distanciamiento, según la cual las personas que no dispongan de acceso a las TIC, o carezcan de las capacidades necesarias para su uso, experimentaran un mayor alejamiento del conocimiento, cosa que aumentará su situación de desventaja sociocultural (Bautista, 2001).

Para paliar esta situación, se está popularizando el desarrollo de políticas para la aceleración de la adopción de estas tecnologías por parte de los ciudadanos. Más allá de disponer del equipamiento de hardware, esta adopción implica el acceso a Internet, así como el desarrollo de las capacidades necesarias para su uso. Este tipo de políticas se fundamentan en la confianza que, de un tiempo a esta parte, se está depositando en las posibilidades de las TIC para luchar contra la exclusión social, también llamada "tecno-optimismo" (Van Winden, 2003).

Según esta opinión, aumentar los niveles de acceso a las TIC puede conllevar una serie de beneficios para el desarrollo de las zonas urbanas, tanto a nivel económico, como social y político: a) beneficios económicos, como el crecimiento del mercado de la electrónica o la inserción laboral de personas en el paro; b) beneficios políticos, en tanto que la implantación del gobierno electrónico puede incrementar la calidad y la frecuencia 
en la producción de políticas, así como estimular la participación civil en la toma de decisiones a nivel local y global; y c) pero sobre todo, se cree que puede ser un instrumento para involucrar a las personas en redes sociales de múltiples tipos que les permitan hacer frente a los problemas y reducir las desigualdades sociales. Por lo tanto, de alguna manera se considera que niveles elevados de adopción de las TIC pueden mejorar la calidad de vida de los ciudadanos/as. El Gráfico 1 representa la multidimensionalidad del concepto de exclusión y el potencial de las TIC en su reparación.

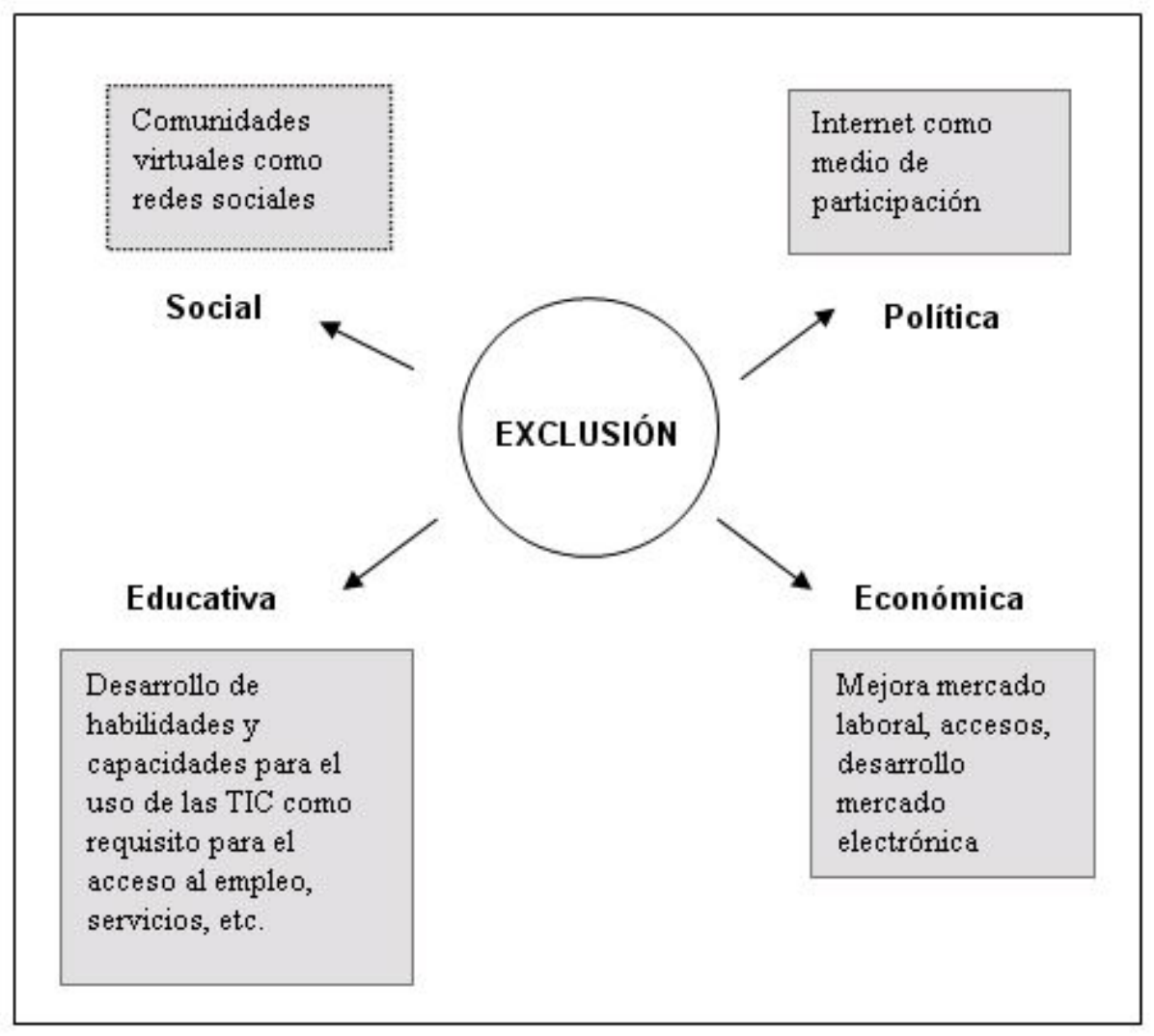

Gráfico 1: Dimensiones del concepto de exclusión social y aportaciones posibles de las TIC a cada una.(adaptación de Van Windem, 2003)

Algunas de las razones por las cuales se considera que las TIC pueden tener un papel importante en la potenciación de la inclusión social, según el Proyecto BEEP (Millard, 2003) son dos principalmente: a) la ubicuidad de las TIC, que las hace indispensables para la participación en la sociedad del conocimiento; y b) la interdependencia existente entre la inclusión social y la e-inclusión, que las hace reforzarse mutuamente. Por otra parte, los objetivos que el Proyecto BEEP propone para luchar contra la exclusión social con la ayuda de las TIC son las siguientes: a) facilitar el acceso "universal” a las TIC; b) mejorar la calidad de vida de grupos vulnerables o en desventaja, explotando el potencial social de las TIC; c) llevar las TIC a las escuelas, donde actúen como impulsoras de formas de aprendizaje innovadoras; d) hacer frente al vacío de conocimientos mejorando la formación continua a través de y sobre las TIC; y e) cooperar contra la fractura digital. Objetivos como estos se desarrollan y materializan en forma de políticas a las que nos dedicamos en el siguiente apartado.

\subsection{Políticas de uso de las TIC para la inclusión social}

El uso de las TIC para la inclusión social implica el facilitar que personas y comunidades hagan un uso lo más provechoso posible de las herramientas y servicios tecnológicos, de modo que les capacite para participar plenamente en la sociedad y les permita controlar el rumbo de sus vidas. Lograr este objetivo, una sociedad de la información y del conocimiento para todos y todas, es una de las prioridades políticas de la Comisión Europea desde la celebración del Consejo Europeo en Lisboa en Marzo del 2000. Así lo demuestran aspectos como las renovaciones del Plan de Acción eEurope[2] y la realización de encuestas específicas sobre einclusión y exclusión social a través del Eurobarómetro[3].

De acuerdo con Van Winden (2003), las políticas para el acceso de todos/as a las TIC deberían, en primer lugar, tener en cuenta los obstáculos para la adopción de las TIC de las poblaciones a las que van dirigidas. 
Según este autor, las políticas más eficientes no son genéricas, si no que se proponen facilitar el acceso a recursos cognitivos, sociales y materiales de grupos específicos con ausencia de acceso a las TIC. Estos grupos se concentran con frecuencia en los barrios marginales de las grandes ciudades, las cuales son además focos importantes de actividades económicas basadas en las TIC.

El informe final del proyecto BEEP (Millard, 2003) expone un conjunto de buenas prácticas para la inclusión social en términos de políticas públicas, de las cuales queremos destacar las siguientes: a) acceso público a las TIC, seleccionando la ubicación más adecuada para la población a la que va dirigido; b) mejora de la interficie del usuario mediante la incorporación del concepto de adaptabilidad; d) inclusión de los grupos objetivo en el diseño de los programas de los cursos; e) realización de los cambios necesarios en la formación de profesores para lograr un uso adecuado de las TIC en las escuelas; y f) inclusión de las TIC en las actividades pre-escolares.

Por su parte, Van Winden (2003) presenta en su estudio cuatro tipos de políticas para la adopción de las TIC: a) la creación de centros de acceso a las TIC para grupos desfavorecidos; b) el acceso gratuito a Internet desde instituciones públicas; c) el acceso gratuito a Internet para todos los ciudadanos en sus hogares; y d) la creación de espacios electrónicos para cada barrio o comunidad de vecinos. Entre los países de la Unión Europea se pueden encontrar diversas aplicaciones estos tipos de políticas. En su mayoría no se dedican a poblaciones de franjas de edad determinadas, si no que se llevan a cabo en programas dirigidos globalmente a poblaciones desfavorecidas, aunque desde acciones concretas para un público infantil, joven, adulto o de la tercera edad[4].

Van Winden (2003) concluye en su estudio que, para conseguir beneficios, las políticas de estímulo al acceso a Internet deberían contemplar los tres tipos de recursos mencionados (materiales, cognitivos y sociales). Así, según el autor es necesario, por una parte, poner a disposición de los potenciales usuarios terminales de Internet para el uso público gratuito, pero esto no es suficiente, y debe complementarse con programas dirigidos específicamente a grupos deficitarios, que estimulen las relaciones comunitarias.

El mismo autor (Van Winden, 2003) realiza unos estudios de caso en tres ciudades comprometidas con el uso de las TIC como medio para hacer frente a la exclusión social: Manchester, La Haya y Rotterdam. Van Winden considera que estas ciudades constituyen un ejemplo del entusiasmo que han despertado las TIC como catalizadoras de cohesión social, de mejoradas formas de participación en la vida política, en el mercado de trabajo, etc. A partir de los estudios de caso realizados, el autor desarrolla un análisis valorativo del tipo de énfasis que ponen las políticas vigentes sobre el uso de las TIC y a que dimensión de la exclusión social, -económica, política, social o educativa-, se refieren[5].

Lo que se observa en todas estas experiencias es que la fractura digital, es decir, la falta de acceso y uso de las TIC por diferentes motivos, es el impedimento principal para el éxito de las políticas de inclusión social comentadas. Diversos estudios muestran que los grupos sociales más débiles, o con mayor riego de exclusión social (desempleados, minorías étnicas, con bajos ingresos, personas con bajo nivel de formación, ancianos, etc.) son los que tienen niveles de acceso y de uso de las TIC más bajos. Por lo tanto, se trata de un problema añadido, o de una nueva dimensión de la exclusión que traspasa todas las anteriores. En consecuencia, se concluye que las políticas deben conducirse, en primer, lugar a favorecer el acceso y el uso de las TIC por parte de estos colectivos y en las áreas más deficitarias. No basta con colocar puntos de acceso a las TIC en cualquier lugar público, puesto que, lo más probable, es que las personas más necesitadas no las utilicen.

En cualquier caso, Van Winden (2003) señala que, a pesar de que el corto tiempo de aplicación de las medidas comentadas no permite extraer conclusiones tajantes, es posible que las expectativas depositadas en el papel de las TIC estén sobrevaloradas. Como mantiene Graham (1999) la tecnología no es un facto independiente, si no que los procesos económicos, sociales, políticos, culturales, institucionales, etc. influyen en la forma en que la tecnología se desarrolla y se aplica. Así, el uso de las TIC puede amplificar el poder político, económico y social de las personas que ya dispongan de él en cierta medida, pero la falta de acceso a las TIC para las personas que no posean este poder, provocará incluso su disminución.

\subsection{Medidas dirigidas a niños/as y jóvenes}

En este apartado nos referimos a programas y experiencias concretas derivadas de las políticas comentadas en el apartado anterior. En este caso, no obstante, nos centramos en medidas dirigidas a niños/as y jóvenes. Para ello, hemos escogido las experiencias presentadas en la tabla siguiente, de entre todas las presentadas en una publicación de la Comisión Europea sobre prácticas de “e-inclusión” en Europa (2001a)[6]. Las experiencias 
se encuentran clasificadas en la tabla según el tipo de actuación en la que hacen más énfasis.

Siguiendo la línea de argumentación precedente, al hablar de medidas dirigidas a colectivos vulnerables menores de edad, nos referiremos a medidas eminentemente preventivas de futuras situaciones de exclusión social. En el caso de las medidas dirigidas a jóvenes, como por ejemplo aquellas orientadas al acceso al empleo, estaremos hablando, en todo caso, de medidas paliativas de situaciones muy germinales exclusión.

\begin{tabular}{|c|c|}
\hline TEMÁTICAS & EXPERIENCIÈS \\
\hline \multirow{3}{*}{$\begin{array}{l}\text { Desarrollo } \\
\text { Comunitario } \\
\text { Local }\end{array}$} & $\begin{array}{l}\text { El Proyecto Ómnia, desarrollado en Cataluña, ofrece puntos informáticos en barrios } \\
\text { desfavorecidos, para que personas en situación de riesgo de exclusión se familiaricen con las } \\
\text { TIC. Los objetivos principales son: a) la inserción laboral: ayudando a las personas a conocer } \\
\text { mejor el mercado laboral a través de la informática; b) la formación en TIC: que contribuye al } \\
\text { alcance de otros objetivos del Proyecto; c) el desarrollo comunitario: mediante la creación de } \\
\text { espacios accesibles para todo el mundo y en especial a personas en situación de riesgo de } \\
\text { exclusión (jóvenes, mujeres, niños y niñas, inmigrantes, etc.). Las actividades incluyen la } \\
\text { creación de redes ciudadanas y lugares de encuentro (reales y virtuales) para organizaciones y } \\
\text { grupos, así como el desarrollo de actividades sobre temas de interés común a través de grupos de } \\
\text { trabajo. El proyecto, también a través de su web, ofrece posibilidades para la interacción a través } \\
\text { de las TIC, incluyendo la realización de videoconferencias. }\end{array}$ \\
\hline & $\begin{array}{l}\text { En Italia la ciudad de Trieste lanzó una serie de proyectos para impulsar la alfabetización en TIC } \\
\text { de jóvenes y mayores indistintamente. La ciudad construyó estructuras y organizó cursos que } \\
\text { implicaran contacto directo entre formadores y participantes. La iniciativa se diseñó de forma que } \\
\text { encajara con los centros recreativos de la misma ciudad. Estos centros, de gran popularidad entre } \\
\text { los jóvenes de Trieste, se equiparon con estaciones multimedia que permitieran a los usuarios, no } \\
\text { solo navegar por Internet, sino producir trabajos creativos. El proyecto "Grandparents and } \\
\text { grandchildren", por otra parte, fue diseñado para familiarizar a los miembros de la comunidad de } \\
\text { más edad con las herramientas para la exploración de Internet. Sus formadores fueron jóvenes } \\
\text { estudiantes voluntarios. Un proyecto similar llamado "Nonn@online" ("Grandparents online") fue } \\
\text { activado por la Comisión Nacional por la Igualdad de Oportunidades. }\end{array}$ \\
\hline & $\begin{array}{l}\text { En Suecia, dentro de la red del Plan Nacional de Acción por los ancianos, "Senior surfing days" } \\
\text { se organizaron proyectos donde jóvenes y personas de la tercera edad pudieran conocerse } \\
\text { mediante las TIC. }\end{array}$ \\
\hline $\begin{array}{l}\text { Desarrollo } \\
\text { Comunitario } \\
\text { local }\end{array}$ & $\begin{array}{l}\text { En España, a través de la iniciativa "Internet para todos", un millón de ciudadanos son objeto de } \\
\text { un programa de cursos de } 15 \text { horas de duración presenciales o virtuales, enfocados al desarrollo } \\
\text { de las capacidades más básicas para el acceso a Internet. Las personas que reciben formación, } \\
\text { son especialmente desempleados y jóvenes en situación de riesgo de exclusión. Los estudiantes } \\
\text { pagan, tan solo, una pequeña cantidad. Los curso están desarrollados por voluntarios de } \\
\text { organizaciones de padres, ancianos, o asociaciones de vecinos. }\end{array}$ \\
\hline \multirow{3}{*}{$\begin{array}{l}\text { Inserción } \\
\text { laboral } \mathbf{y} \\
\text { empleabilidad }\end{array}$} & $\begin{array}{l}\text { El proyecto “Stepping Stones” desarrollado en Irlanda se propone mejorar las capacidades y } \\
\text { habilidades personales y educativas de jóvenes desempleados en situación de desventaja. El } \\
\text { Waterford Youth Committee y el Princes Trust Pembrokeshire se encargan del proyecto. Este } \\
\text { ofrece cursos de informática, de construcción de equipos, primeros auxilios, proyectos } \\
\text { comunitarios y medioambientales, un programa de experiencia de trabajo y un título nacional de } \\
\text { formación sobre capacidades básicas. La formación profesional intensiva abarca la construcción } \\
\text { de equipos, la formación del liderazgo, formación y titulación en el uso de TIC, etc. con el } \\
\text { objetivo de mejorar las posibilidades de empleo, o empleabilidad de los participantes }\end{array}$ \\
\hline & $\begin{array}{l}\text { En Italia, la red de centros de información para los jóvenes "Centri Informa Giovani59", } \\
\text { constituida por más de } 500 \text { centros en grandes y pequeñas ciudades, ofrecen acceso a } \\
\text { información sobre empleo, desarrollo y creación de empresas, TIC, etc. }\end{array}$ \\
\hline & $\begin{array}{l}\text { En la misma línea, dos programas de formación llevados a cabo en el sur de Italia se dedican a } \\
\text { jóvenes desempleados, centrándose en las capacidades para el uso de las TIC y el aprendizaje del } \\
\text { inglés. El Programa Knowledge, para trabajadores y personas desempleadas, se dedica a la } \\
\text { formación de expertos en TIC. }\end{array}$ \\
\hline Acce & $\begin{array}{l}\text { En el Reino Unido, el proyecto New Deal para los jóvenes, desarrollado a través del Employment } \\
\text { Service, está dirigido a personas que necesitan un empleo e incorpora, de forma gratuita, las } \\
\text { entrevistas y el tipo de formación más adecuada. Este programa contribuye a resolver todo tipo }\end{array}$ \\
\hline
\end{tabular}




\begin{tabular}{|c|c|}
\hline $\begin{array}{l}\text { TIC y } \\
\text { formación } \\
\text { sobre TIC }\end{array}$ & $\begin{array}{l}\text { de cuestiones a las personas que buscan trabajo: 1) acceso; a través de centros de aprendizaje } \\
\text { mediante TIC, pueden formarse y conocer de todo lo que supone buscar trabajo-; 2) PCs gratis o } \\
\text { subvencionados para su propio uso y control; 3) formación; gratuita o subvencionada en } 50000 \\
\text { centros de aprendizaje virtual. Ésta experiencia mostró el potencial de la publicidad local vía } \\
\text { televisión o radio, así como de la vinculación a las iniciativas ya existentes en la comunidad, que } \\
\text { hubieran probado ser de ayuda, o que tuvieran una buena imagen. }\end{array}$ \\
\hline $\begin{array}{l}\text { Acceso a las } \\
\text { TIC y } \\
\text { formación } \\
\text { sobre TIC }\end{array}$ & $\begin{array}{l}\text { En Finlandia, existen facilidades para el cuidado de niños y niñas en edad preescolar, por parte } \\
\text { de las administraciones municipales, que proveen de materiales informáticos para jugar y } \\
\text { aprender. }\end{array}$ \\
\hline \multirow{4}{*}{$\begin{array}{l}\text { Sensibilización } \\
\text { sobre el acceso } \\
\text { y uso de las } \\
\text { TIC }\end{array}$} & $\begin{array}{l}\text { En lo que se refiere a las medidas de concienciación sobre el uso de las TIC, en el Reino Unido } \\
\text { incluyen anuncios de TV sobre la línea de ayuda online "Learndirect” y otros cursos, basados o } \\
\text { no en las TIC. A través de Centros de Aprendizaje virtuales y las promociones locales y } \\
\text { nacionales, así como programas de televisión, se da promoción a programas de acceso a } \\
\text { ordenadores gratis o subvencionados para los grupos objetivo, llamados “Computers within } \\
\text { Reach” y “Wired Up Communities”. A través de la página web, el gobierno selecciona a } 50000 \\
\text { personas que recibirán cursos gratuitos de formación sobre TIC. }\end{array}$ \\
\hline & $\begin{array}{l}\text { En Bélgica, el Ministerio de Asuntos Exteriores organizó el "RoadShow 2000-Tous sur le net”, } \\
\text { una campaña de sensibilización en la que tomaron parte todos los grandes núcleos de ciudades y } \\
\text { pueblos. El objetivo fue concienciar sobre la importancia de las TIC, estimular a los propietarios } \\
\text { de PCs a conectarse a Internet, animar a las empresas (especialmente a las pequeñas y medianas } \\
\text { empresas) a crear redes y a despertar el interés de los jóvenes por las TIC. Esto se complementó } \\
\text { con una guía de Internet, con información práctica sobre como configurar una conexión de red, } \\
\text { Internet y el correo electrónico, navegadores, otros programas relacionados, etc. }\end{array}$ \\
\hline & $\begin{array}{l}\text { “Fritt Fr@m” es el nombre de una campaña informativa llevada a cabo en Suecia, la cual } \\
\text { promueve la idea de una sociedad de la información para todos/as. Los agentes responsables son } \\
\text { el Swedish Handicap Institute y las organizaciones más importantes para personas con } \\
\text { discapacidades. Las actividades de la campaña están dirigidas a diseñadores, pero también a } \\
\text { políticos y a todo el público en general. }\end{array}$ \\
\hline & $\begin{array}{l}\text { En Francia, la red de información, "Réseau Information Jeunesse", consta de } 600 \text { "points } \\
\text { cyberjeunes" que están siendo desarrollados con acceso a Internet y dos formadores por punto. }\end{array}$ \\
\hline
\end{tabular}

Tabla 1. Clasificación de experiencias

\section{Comentarios finales}

Después de investigar y analizar las políticas y programas de estímulo al acceso y uso de las TIC por parte de los jóvenes, y para terminar, nos gustaría plantear dos reflexiones.

En primer lugar, reconocemos y apoyamos el valor de estas acciones para favorecer la inclusión social, especialmente como medidas preventivas de futuras situaciones de exclusión, en el caso de las personas más jóvenes. Dentro de estas, consideramos positiva la incorporación de las TIC, por diversos motivos: por una parte, el acceso y el uso de las TIC tienen valor en si mismos al paliar o, al menos contrarrestar, el desarrollo de la fractura digital; por otra parte, el potencial educativo y el efecto motivador de las TIC entre los más jóvenes, favorecen el alcance de los objetivos formativos que se hayan propuesto.

No obstante, consideramos que, en efecto, puede estarse depositando una confianza excesiva en las TIC como procedimiento para remediar situaciones de exclusión social. A pesar de las posibilidades que ofrecen, estas acciones deben ir acompañadas de otras medidas políticas de tipo económico, educativo y social, que contribuyan, desde diferentes sectores, a promover la igualdad de oportunidades de las personas en situación de desventaja social. Un uso exclusivo de las TIC en las políticas de inclusión, solo puede resultar estéril.

Por esta razón, creemos que es importante aclarar que las medidas presentadas no están llamadas a sustituir, si no a complementar, otro tipo de acciones en el ámbito del bienestar social. La promesa de las TIC como camino a la igualdad, puede ser peligrosa si se olvida que el uso de las tecnologías también depende de otros factores vitales, que deben ser atendidos. 
AREA, M. (coord) (2001) Educar en la Sociedad de la Información. Col. Aprender a ser. Bilbao: Desclée de Brouwer.

ARRIBA, A. (2002) El concepto de exclusión en política social. Unidad de Políticas Comparadas. Consejo Superior de Investigaciones Científicas (CSIC). Documento de trabajo [En línea: http://www.iesam.csic.es/doctrab2/dt-0201.pdf] [Fecha de consulta: octubre de 2004]

BAUTISTA, A. (2001)Desigualdades sociales, nuevas tecnologías y política educativa, en Area, M. (coord) Educar en la Sociedad de la Información. Col. Aprender a ser. Bilbao: Desclée de Brouwer, 179-213.

BECTA (2001) The digital divide: a discussion paper. Prepared for the DIES by the Evidence Team, BECTA [En línea: http://www.becta.org.uk/research/reports/docs/digitaldivide.pdf] [Fecha de consulta: enero de 2002]

BHALLA, A. y LAPEYRE, P. (1997) Social exclusion: towards an analytical and operational framework, Development and Change, 28(3), 413-434.

CASTEL, R. (1997) La metamorfosis de la cuestión social. Una crónica del salariado. Buenos Aires, Paidós.

CASTELLS, M. (2001) La era de la información. Economía, sociedad y cultura. Mèxic: Ed. Siglo XXI. Vol. II.

COMISIÓN EUROPEA (2001a) E-Inclusion. The Information Society's potential for social inclusion in Europe. E-inclusion practices. Documento de trabajo. [En línea: $<$ http://europa.eu.int/comm/employment_social/socdial/info_soc/esdis/eincl_2] links.htm> [Fecha de consulta: febrero de 2002]

COMISIÓN EUROPEA (2001b) E-Inclusion. The Information Society’s potential for social inclusion in Europe. Documento de trabajo. [En línea: http://europa.eu.int/comm/employment_social/socdial/info_soc/esdis/eincl_2links.htm [Fecha de consulta: febrero de 2002]

COMISIÓN EUROPEA (2002) eEurope 2005: An information society for all. Documento de trabajo. [En línea:

http://europa.eu.int/information_society/eeurope/2002/news_library/documents/eeurope2005/eeurope2005_es.pdf ] [Fecha de consulta: septiembre de 2002]

COMISIÓN EUROPEA (2003) Building the knowledge society: social and human capital interactions. Documento de trabajo. [En línea: http://europa.eu.int/comm/employment_social/knowledge_society/shc_en.pdf] [Fecha de consulta: septiembre de 2002]

GRAHAM, S. (1999) Towards urban cyberspace planning: grounding the global through urban telematics policy and planning, en Downy, J. y McGuigan, J (eds.), Technocities, Sage, London.

LA PARRA, D. y TORTOSA, J.M. (2002) Procesos de exclusión social: redes que dan protección e inclusiones desiguales. Revista del Ministerio de Trabajo y Asuntos Sociales, 35. [En línea: $<$ http://www.mtas.es/publica/revista/numeros/35/estudio3.pdf $>$ ] [Fecha de consulta: noviembre de 2004]

LÉVY, P. (1998) La cibercultura, el segon diluvi? Barcelona: Proa

MARCO, M. (2000) Consideraciones en torno al concepto de exclusión social. Revista Acciones e Investigaciones Sociales, $\quad$ [En 9-21. http://wzar.unizar.es/acad/fac/eues/REVISTA_AIS/11_AIS/AIS_11(03).pdf] [[Fecha de consulta: octubre de 2000]

MILLARD, J. (Ed.) (2003) BEEP Project Final Report. Best eEurope Practices. Bruselas y Dinamarca, Comisión Europea y Danish Technological Institute [En línea: <http://www.beep-eu.org/>] [Fecha de consulta: octubre de 2004]

INTERFACE \& DANISH CENTRE (2001) Addressing Social Exclusion via the e-Learning Model. Annex 
5.1. Workpackage 5. SDV-NETJOB Project. Promoting Accessible Social Dialogue and Innovative Training Practices: Towards an Information Society for All [En línea: http://www.socialdialogue.net] [Fecha de consulta: noviembre de 2004]

SUBIRATS, J. (dir.) (2004) Pobreza y exclusión social. Un análisis de la realidad española y europea. Colección Estudios Sociales, 16. Barcelona, Fundación La Caixa. [En línea: $<$ http://www.estudios.lacaixa.es>] [Fecha de consulta: diciembre de 2004]

TRANSIT PROJECTES (2004) E-learning hacia la inclusión social. Barcelona. [En línea: <http://www.el4ei.net/first/charter\%20-\%20carta/charter_E-learning_hacia_inclusion_social.pdf>] [Fecha de consulta: noviembre de 2004]

VAN WINDEN, W. (2003) Essays on urban ICT policies. Tinbergen Institute Research Series, 328. [En línea: http://www.kcgs.nl/kcgs/show/id=195949/contentid=4046] [Fecha de consulta: noviembre de 2004]

WELLMAN, B.; BOASE, J.; CHEN, W. (2002) The networked nature of community: online and offline. IT\&Society, 1 (1) 151-165.

\section{Notas:}

[1] En cualquier caso como comenta Subirats (2004) el uso del término exclusión, en el contexto de las políticas sociales en Europa, no se corresponde con su significado estricto (deportación o expulsión de una comunidad, reclusión, etc.). Si no que se refiere más bien a procesos de vulnerabilidad, de desconexión social, de pérdida de lazos sociales y familiares que, generados por una combinación de factores y situaciones de desigualdad, marginación, etc. Siguiendo el razonamiento de Subirats, el concepto de vulnerabilidad enmarca aquellas situaciones de "equilibrio social precario" que pueden agravarse por factores añadidos de marginación o desigualdad, pudiendo desembocar en procesos de exclusión.

\section{[2] http://europa.eu.int/information_society/eeurope/2005/all_about/action_plan/text_en.htm}

[3] Algunas de las áreas elegibles para la aplicación de políticas según la propuesta de la Comisión Europea del año 2001 son las siguientes: a) explotar el potencial de la sociedad de la información para las personas en situación de desventaja: generando contenidos online adecuados, impulsando las comunidades locales a través de servicios y redes online, haciendo posibles las oportunidades de trabajo a través de las TIC; b) eliminar las barreras en la sociedad de la información: despertando la conciencia de las oportunidades que ofrece, haciendo las TIC disponibles y asequibles mediante la creación de puntos de acceso a Internet públicos, incentivando el acceso individual a las TIC, la inversión en infraestructuras de TIC en las regiones más desfavorecidas, y implicando el uso del móvil y la televisión digital para la e-inclusión, promoviendo la alfabetización digital a través del e-learning y haciendo frente a las barreras técnicas para las personas con alguna discapacidad

[4] Por ejemplo, en Manchester se encuentran los “electronic village halls” (EVH's) los cuales ofrecen acceso a Internet gratuito, pero además programas formativos y educativos a muy bajo coste. De esta forma, se combina la aportación de recursos sociales, cognitivos y materiales a los/las participantes. Se tratade una iniciativa de la administración pública local, que parte de la idea que el acceso a las TIC no solopuede reducir el desempleo y la falta de formación, si no que además es un medio para reducir la falta de profesionales en el sector de las TIC.

Helsinki cuenta con una biblioteca pública en el centro de la ciudad: The Cable Book Library. Se trata de un centro con los servicios habituales de acceso a documentación bibliográfica diversa pero, además, ofrece 20 estaciones de trabajo equipadas con ordenadores y acceso gratuito a Internet. En este caso, aunque la iniciativa va dirigida de forma prioritaria a grupos desfavorecidos, en la práctica, la mayor parte de personas que la utilizan tienen ya niveles altos de alfabetización digital, como por ejemplo estudiantes, turistas o jóvenes. Esta iniciativa carece del componente educativo o formativo y, en consecuencia, fracasa en el objetivo de favorecer el desarrollo de recursos cognitivos.

En la ciudad de La Haya se ha implantado el proyecto Residentie.net, el cual se propone que todos los habitantes de la ciudad tengan acceso gratuito a Internet. En el caso de aquellas personas que no disponen de ordenadores, el acceso a Internet se consigue a través del televisor. Además, el proyecto cuenta con un portal 
web en el cual se puede encontrar todo tipo de información gubernamental, periódicos locales, información turística, eventos locales, tráfico, transporte público, etc. La finalidad última de este proyecto es involucrar al mayor número posible de ciudadanos en procesos de toma de decisión a través de las TIC. Si bien, el Residentie.net, carece de componente educativo y por lo tanto no proporciona recursos cognitivos, si favorece la creación de comunidades virtuales locales y por lo tanto contribuye a la construcción de recursos sociales.

Por último, una experiencia ilustrativa del último tipo de política mencionado es ECity desarrollado en Eindhoven. El principal objetivo de este proyecto es crear las condiciones necesarias para la aceleración de la comercialización de nuevos servicios y productos. Este proyecto estimula el dominio, el uso y el desarrollo de capacidades para el uso de las TIC, contribuyendo a la puesta en práctica de los recursos cognitivos, materiales y sociales de la población. Los recursos sociales se fortalecen a través de la creación de comunidades virtuales, mientras los recursos materiales y los cognitivos se potencian mediante la facilitación de acceso a equipos informáticos y cursos de formación a precios muy económicos o totalmente gratuitos.

[5] Entre las políticas dirigidas a paliar la dimensión social de las situaciones de exclusión, las TIC se contemplan como generadoras de nuevas conexiones y relaciones en áreas desfavorecidas, sustentándose en la idea que las herramientas de comunicación a través de Internet facilitan la interacción social entre personas más o menos distantes. En este sentido, se cree que las TIC pueden estimular la integración y el desarrollo de grupos desplazados y reducir el aislamiento de grupos con pocas posibilidades de movilidad, como las minorías étnicas, las personas discapacitadas, los ancianos o las personas que se encuentran en prisión, las personas enfermas, etc. Sin embargo, entre los casos analizados por Van Winden (2003) se encontraron pocas evidencias del hecho que Internet promoviera la formación de redes sociales diversas. Más bien, los indicios apuntaban a la especialización de las redes de grupos de iguales a través de Internet.

En el caso de las medidas gubernamentales que contemplan la dimensión política de la exclusión, el papel de las TIC es estimular la participación ciudadana. Por ejemplo, a través de páginas web que incorporen foros de discusión sobre cuestiones relacionadas con la toma de decisiones a escala municipal o regional. Se pueden crear plataformas tecnológicas de tipos diversos, en las cuales los ciudadanos y ciudadanas puedan aportar opiniones e ideas y exponer sus quejas. La conclusión de Van Winden (2003) a este respecto es que, a pesar de reconocer la existencia iniciativas de promoción de la participación civil en los procesos de toma de decisión, no se encontró evidencia alguna de que los grupos excluidos socialmente, o en riesgo de exclusión, utilizaran ese tipo de facilidades. Por el contrario, parece lógico pensar que estos grupos se desilusionaran al intentar tomar parte de estas iniciativas, entre otras cosas por no disponer de acceso a Internet.

En este sentido, la potenciación de la participación política a través de las TIC, puede incluso provocar una disminución de los niveles de participación originales de los grupos excluidos socialmente.

Finalmente, la dimensión económica y educativa de la exclusión se encuentran muy interrelacionadas pues son interdependientes. Se cree que, dada la escasez de personal preparado para trabajar en la industria de las TIC, las personas excluidas del ámbito laboral pueden mejorar sus oportunidades de incorporación al trabajo si adquieren conocimientos y desarrollan capacidades y habilidades relacionadas con las TIC. Incluso las personas con conocimientos básicos sobre el uso de las TIC pueden mejorar notablemente sus oportunidades en el mercado laboral, si paralelamente se da un desarrollo de ciertas capacidades cognitivas y sociales. Por lo tanto, las políticas para el acceso al empleo, las educativas o de formación deberían tener en cuenta el papel que pueden jugar las TIC para el acceso al empleo o para futuras oportunidades de formación. Además, mediante la teleformación y el teletrabajo, las TIC pueden ser una alternativa, tanto formativa como laboral, para los grupos con poca o nula movilidad, pudiendo repercutir además en la mejora de la autoestima y la ampliación de las redes sociales de estos grupos. Dado lo reciente de este tipo de iniciativas, resulta casi imposible hablar de resultados. A pesar de ello, Van Winden (2003) encontró cierta evidencia de personas que se habían reincorporado al trabajo, gracias a los aprendizajes construidos en torno a las TIC.

[6] Esta colección de buenas prácticas está basada en las aportaciones del Grupo ESDIS (http://europa.eu.int/comm/employment_social/knowledge_society/esdis_en.htm), sobre los Planes de Acción Nacionales para la inclusión social, entre otros documentos (http://europa.eu.int/comm/employment_social/soc-prot/soc-incl/index_en.htm) 
Lourdes Pérez Sánchez

Correos electrónicos por orden: fird@usal.es

o bien lopesan@usal.es 